OPEN

SUBJECT AREAS:

PARASITOLOGY

IMMUNITY

MICROBIAL GENETICS

MOLECULAR EVOLUTION

Received

19 December 2011

Accepted

2 March 2012

Published

30 March 2012

Correspondence and requests for materials should be addressed to

W.P. (wqpan0912@ yahoo.com.cn)

\section{Having a pair: the key to immune evasion for the diploid pathogen Schistosoma japonicum}

\author{
Xindong X ${ }^{1}$, Jun Sun' , Jingiing Zhang' ${ }^{1}$, Dianne Wellems ${ }^{1,2}$, Xiaoxing Qing' ${ }^{1}$, Thomas McCutchan ${ }^{2}$ \\ \& Weiqing Pan $^{1,2}$
}

'Institute for Infectious Diseases and Vaccine Development, Tongii University School of Medicine, 1239 Siping Road, Shanghai 200092, PR China, ${ }^{2}$ Department of Pathogen Biology, Second Military Medical University, 800 Xiang Yin Road, Shanghai 200433, PR China.

Schistosomes, unlike malaria parasites, are in their diploid stage when targeted by the human immune system. Diploids can be either homozygous or heterozygous. The difference has profound significance for developing immunity and yet has not previously been addressed. We examined the implications of zygosity on immunity to a diploid pathogen, Schistosoma japonicum and showed that the diploid state, and its associated heterozygous advantage, significantly affects the outcome of attack by the immune system and the accumulation of antigenic diversity in the parasite population. We demonstrate here that diploidy provides a novel means of immune evasion for diploid pathogens.

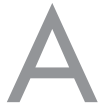

lthough both Schistosomes and malaria parasites have sexual cycles, the Schistosomes are in the diploid stages of their cycle when targeted by the human immune response, while malaria parasites are in their haploid stages. Since antigenic variation appears to be a major foil in the fight against infectious disease, it is important to consider the basic differences between haploid and diploid organisms as they relate both to immune evasion and to the accumulation of antigenic diversity in the population.

Schistosomes are diploid pathogens that cause the second most deadly human parasitic disease after malaria. Vaccination, either alone or in combination with drug treatment, represents our best long-term hope for controlling schistosomiasis ${ }^{1-3}$, but despite decades of research and development we still do not have an effective vaccine. Recent attempts to develop a vaccine against Schistosoma mansoni, using tegument proteins (Sm-TSP-2 and Sm29) to stimulate an immune response, have met with some success ${ }^{4,5}$. We are, however, still perplexed by the fact that vaccination with Sj-TSP-2 and Sj29, the orthologue to Sm-TSP-2 and Sm29 $9^{6-8}$, kills only a small percentage of the parasites. One likely reason for the meager level of success appears to involve variations of the immunogens $s^{7,9}$. To date, most information about the antigenic variation of pathogens derives from the study of organisms whose life cycle is predominantly haploid ${ }^{10-12}$. One important question is whether our knowledge of the control of haploid organisms is, in fact, directly applicable to the development of diploid organisms. Diploids can be either homozygous or heterozygous. Scientists often do not agree about the relative advantages of the diploid and haploid states, although most do agree that the so-called "heterozygous advantage" benefits diploid organisms under certain circumstances (e.g. in the sickle cell genotype) and thereby affects the survival of the organism.

Our study focuses on the fundamental biological impact of diploidy on the battle between the immune system and diploid pathogens.

\section{Results}

Expression of the Sj29 gene. We first isolated and sequenced the entire Sj29 gene. A comparison of its gene sequence with cDNA sequences indicated that the exon-intron structure of the Sj29 is identical to that of Sm29 (Supplementary Fig. 1A). We then established that both copies of the Sj29 genes were expressed in individual worms by sequencing $\mathrm{cDNA}$ from each of six. Four worms were heterozygous, containing two different $\mathrm{Sj} 29$ genes and transcripts (Supplementary Fig. 1B). Only a single type of Sj29 gene and cDNA sequence was found in either of the other two worms, and it was concluded that they were homozygous for the gene (Supplementary Fig. 1C). 
Sequence polymorphism. We obtained total 268 full-length sequences of the $\mathrm{Sj} 29$ gene from 134 individual worms derived from a single field-isolate of $S$. japonicum cercariae. From these, 37 different allelic forms were identified (Supplementary Table 1). Two types of analysis were done to establish the relationship among the alleles: neighbor-joining analysis (Mega 4) indicated that these could be resolved into distinct groups (Fig. 1A); cladistic analysis (MacClade) indicated that most of the worms derived from a small number of lineages or clades (Fig. 1B). Here we refer to three groups as clades A, B and $\mathrm{M}$ ( $\mathrm{a}$ mixture of alleles that could not be definitively included in clades A or B). We will refer to the latter as "clade" M, although it is likely to represent more than one lineage, and avoid reference to it in situations where direct lineage is a factor in interpreting results.

Immunization and challenge. We chose the most common allele from each clade for subsequent immunological analysis: clade A, Sj29-2; clade B, Sj29-4 and "clade" $\mathrm{M}, \mathrm{Sj} 29-20$. These alleles represent $86.0 \%, 83.6 \%$ and $49.2 \%$ of their individual clade, respectively. The recombinant proteins were produced in Pichia pastoris for immunization experiments. Mice ( $n=10$ per group) were immunized with the antigens formulated with adjuvant ISA $70 \mathrm{M}$ or the adjuvant alone and PBS as controls and challenged with 40 cercariae. Survival adult worms from each group were recovered six weeks following challenge and genotyped for Sj29 gene. The surviving worms in individual groups were categorized as homozygotes (AA, BB) and heterozygotes $(\mathrm{AB}, \mathrm{AM}, \mathrm{BM}$ and $M M)$. We will henceforth refer to worms carrying both copies of the $\mathrm{Sj} 29$ gene from the same lineage as homozygous.

Vaccination with $\mathrm{Sj} 29-2$ provided significant protection against the AA genotype. The results showed that only 4 worms that were homozygous for the A clade survived in the vaccinated group, representing $2.3 \%$ of total survivors, while 34 AA homozygotes survived in the adjuvant control group, representing $13.7 \%$ of total survivors (Fig. 2A, Supplementary Table 2). This indicated $88.2 \%$ reduction in AA worm burden. Vaccination with Sj29-2 provided no significant protection against heterozygous A genotypes with 33 surviving worms (37.1\% of total worms). The experiment was repeated with like results (Fig. 2B).

We then tried the same experiment using a Clade B antigen, Sj29-4. The number of Sj29-4 targeted BB homozygous worms was again significantly reduced (Fig. 2C). Hence, immunization with single proteins from either of the two major clades resulted in significant protection from infection by homozygotes of their respective lineages (AA or $\mathrm{BB})$. On the other hand, exposure to either of the proteins had no effect on the protection of heterozygote infection. We also exposed mice to the Sj29-20 protein "clade" M. The results were consistent with those shown for other clades, but the number of homozygotes in the field-collected challenge isolate was too small to lend statistical support for the idea of clade specific protection (Supplementary Fig. 2).

We immunized mice with a mixture of Sj29-2, Sj29-4 and Sj29-20 antigens and challenged these mice with field-collected

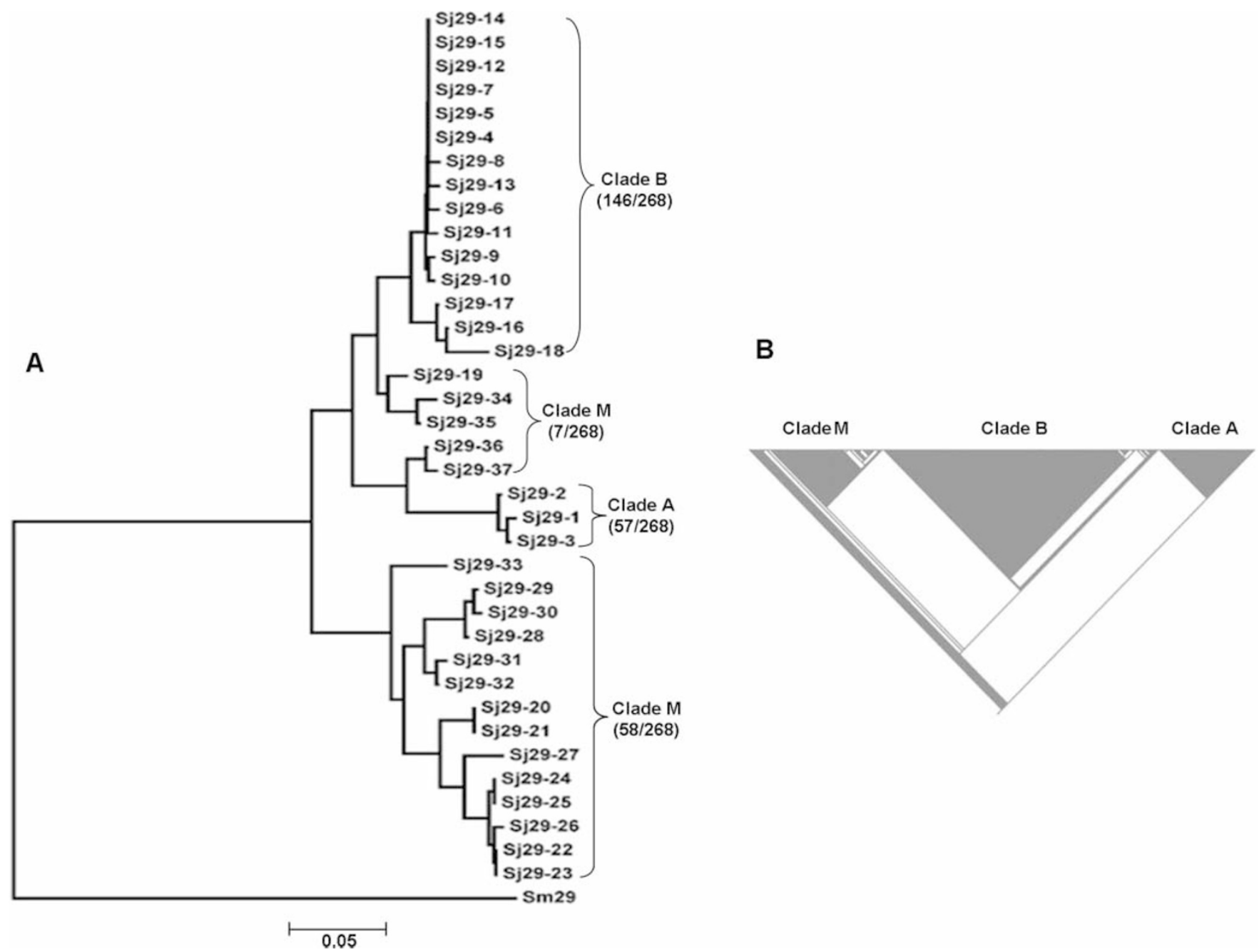

Figure $1 \mid$ Analysis of Sj29 sequence. (A) Neighbor-joining tree of Sj29 alleles and Sm29 (AF029222) based on $p$, the proportion of amino acid difference was generated by using Mega 4. (B) Cladogram of Sj29. 

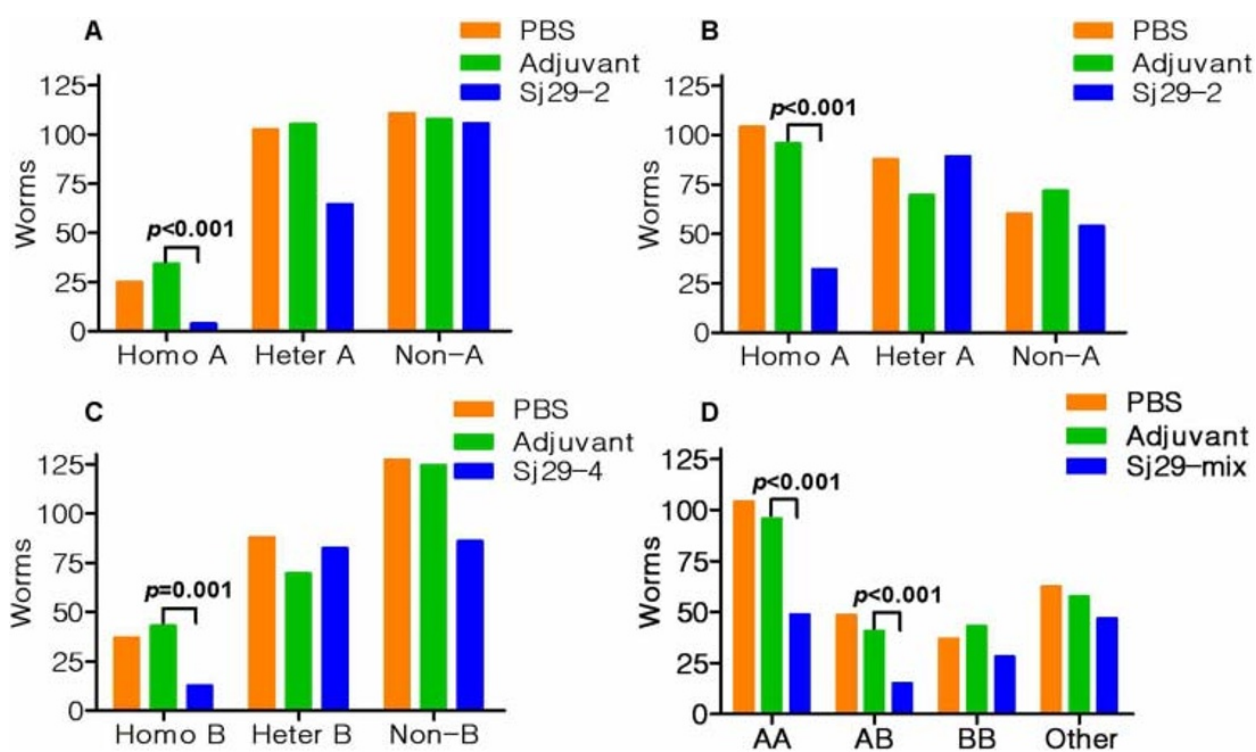

Figure 2 Comparasion of the distribution of homozygotes and heterozygotes in immunized and control groups. Mice (10 per group) were immunized with the antigens (Sj29-2, Sj29-4 or Sj29-mix) adjuvanted by ISA $70 \mathrm{M}$, the adjuvant alone and PBS, respectively and challenged with cercariae. The survival worms from individual group were pooled for genotyping of Sj29 gene. The survival worms from were categorized as homozygotes (Homo-A, Homo-B), heterozygotes (Heter-A, Heter-B and AB) and others(Non-A, Non-B). The number of the surviving worms with various categorized genotypes in the immunization groups were compared to that in the control groups exception of the Sj29-mix group (D) where the data was converted before statistic analysis (see "Statistic Anaysis" in Method section). (A) Sj29-2 immunized group of the first experiment. (B) Sj29-2 immunized group of the second experiment. (C) Sj29-4 immunized group of the second experiment. (D) Sj29 multiple antigens immunized group.

parasites. This greatly enhanced the vaccine's protective effect; it resulted in a $41.4 \%$ reduction in the overall worm burden in comparison to $26.2 \%, 23.6 \%$ and $14.8 \%$ reduction observed when using individual antigen for immunization (Supplementary Fig. $3 \mathrm{~A})$. We then determined the $\mathrm{Sj} 29$ genotype of surviving worms (Supplementary Table 2). Significantly, immunization with the mixture significantly affected the survival of $A B$ heterozygotes, along with the homozygotes (AA) (Fig. 2D). The presence of antibody to both of the expressed alleles appears connected with protection (Supplementary Fig. 4). Moreover, immunization with a mixture of antigens reduced the number of eggs in the liver by 48.8\% (Supplementary Fig. 3B).

Infection by a clonal isolate. To control the genotypical complexity of the infectious agent, we cloned parasite lines from the fieldcollected isolate, as shown in Fig. 3A. Snails were infected with single miracidia. Then cercariae from snails were paired to infect rabbits and adult worms from each rabbit were genotyped. One pair showed only 2 alleles (i.e. Sj29-4/Sj29-4 homozygous female worms and Sj29-2/Sj29-4 heterozygous male worms). This clonal
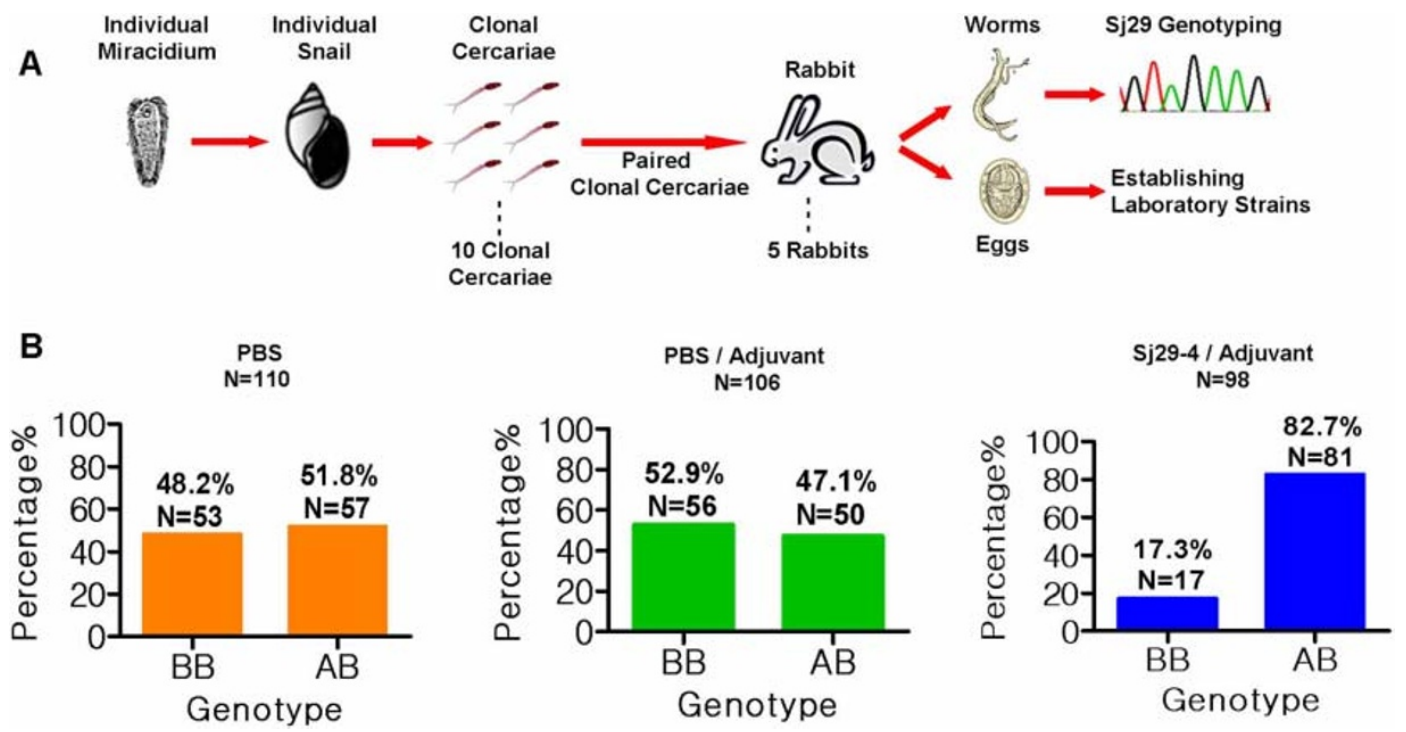

Figure 3 Establishment and infection of clonal parasites. (A) Establishment of clonal parasites. Snails were infected with single miracidia. Then cercariae from snails were paired to infect rabbits and adult worms from each rabbit were genotyped. One pair showed only 2 alleles (i.e. Sj29-4/Sj29-4 homozygous female worms and Sj29-2/Sj29-4 heterozygous male worms). This clonal parasite of S. japonicum was designated Sj6M8. Eggs from this line were collected to establish clonal parasites. (B) Comparasion of the distribution of homozygotes and heterozygotes in Sj29-4 immunized and control groups infected with clonal parasites. $\mathrm{N}$ : the number of sequenced survival worms. 
parasite of S. japonicum was designated Sj6M8. Eggs from this line were collected to establish clonal parasites. Mice were immunized with Sj29-4 recombinant protein (clade B) and then challenged with the clonal line Sj6M8. This resulted in a $43.1 \%$ reduction in worm burden and a $53.6 \%$ reduction of eggs in the liver (Supplementary Fig. 5). Of the survivors in $\mathrm{Sj} 29-4$ immunized group, $82.7 \%$ were $A B$ heterozygotes. In contrast, the ratio of $\mathrm{BB}$ homozygotes and $\mathrm{AB}$ heterozygotes in the control groups was approximately equal (Fig. 3B). Hence, exposure to the clade B antigen resulted only in significant protection against the $\mathrm{BB}$ homozygote. Importantly, the greater extent of overall protection can be attributed to the relatively greater representation of $\mathrm{BB}$ homozygotes in the clonal population. This, as well as data shown in Fig. 2, suggest that minor sequence differences seen within a single Sj29 clade are not the result of immune selection and do not subvert the protective response.

\section{Discussion}

The response of diploid organisms to selective pressure is fundamentally different from that of haploids. This difference is evident in the response of the immune system to the tegument protein Sj29 and the progression of parasitic infection in immunized animals. Here we identified 37 different Sj29 alleles among 268 sequences. The ratio of non-synonymous to synonymous substitutions ( $d \mathrm{~N} / d \mathrm{~S})$ in these variant forms, however, shows no indication of selective pressure on the protein sequence (Supplementary Table 3). Consistent with this result, the alignment of the $\mathrm{Sj} 29$ sequences indicated that the vast majority of sequences fit into distinct lineages with a low degree of homoplasy (sequence similarities that result from convergence rather than ancestral relationships). This would further indicate a lack of immune selection. In contrast, investigations of the diversity of blood stage $P$. falciparum antigens indicated both selective pressure and a high degree of homoplasy ${ }^{13,14}$. Do these observations indicate a flaw in the algorithms meant to identify selective pressure, or that diploid organisms can evade immune response in ways that these formulas do not detect?

We selected one representative protein from each of three lineages and studied the immunological response they elicited. Immunization with a single protein provided significant protection only against the development of Schistosomes that were homozygous for the same allele. A greater number of Schistosomes were killed when immunized mice were challenged with a clonal-isolate than a field-isolate. The percentage of clade-specific homozygotes killed, however, was the same regardless of the source of challenge. This addresses two issues: 1) the fact that same percentage of homozygous worms evaded the immune response regardless of their numbers in the challenge population indicated that their survival did not stem from an inadequate, adaptive-immune response. Solely focusing on a means to increase the potency of the adaptive immune response (e.g., using different adjuvants) may not, therefore, lead to a more effective response; other factors appear to be involved in parasite survival. 2) The heterozygous worms have an advantage over their homozygous counterparts in certain natural situations. This advantage plays a role in preserving genetic diversity in the population. With regard to the latter, a predictive model suggests that although the percentage of each $\mathrm{Sj} 29$ clade within the population would rise and fall, none would become extinct (Supplementary Fig. 6).

Immunization with a mixture of proteins representing three clades, followed by challenge with a field-isolate, supported the concept that products of both alleles need to be recognized by the immune system for a protective response to occur. In this experiment the survival of both homozygotes and heterozygotes was affected. The protection elicited by the mixture of all three proteins approached the sum of the protections offered by them individually. This indicates that the separation of immunogenic alleles according to their clade has immunological relevance. Clearly, a random selection of antigens for immunization would not have had an additive effect on protection.

To date, the accepted approach to preventing parasitic infection by means of stimulating the immune response does not take into consideration whether the pathogen under assault is haploid or diploid. This study is the first to emphasize the importance of zygosity on the immune response to diploid parasites.

\section{Methods}

Parasites and animals. A field-collected isolate of S. japonicum from Guichi County, Anhui Province, China was used in all the experiments. Female 6-8 week old BALB/c mice were obtained from the Songjiang Animal Facility of the Chinese Academy of Sciences of Shanghai. To establish clonal parasites, snails were individually exposed to one miracidia. Single snail-derived clonal cercariae were collected. Rabbits were infected with different cercaria pairs. The adult worms were genotyped and their eggs were used for establishing labaratory lines. All procedures performed on animals within this study were conducted in accordance with and by approval of the Internal Review Board of Tongji University School of Medicine.

Allelic expression analysis. To estimate whether both the maternal and paternal alleles of Sj29 were expressed, single worm was cut into two parts for genomic DNA and total RNA extraction. Genomic DNA, cDNA and RNA (negative control) were used as PCR templates. The sixth exon with 180 bp was amplified by PCR. Sequence chromatogram traces of genomic DNA-amplified products were used to determine homozygote or heterozygote. The chromatogram traces of SNP site showed double peaks in heterozygote but single peak in homozygote. We compared the

chromatogram traces of SNP site of cDNA with genomic DNA to estimate whether the maternal and paternal alleles of $\mathrm{Sj} 29$ were expressed.

Characterization of SNPs in Sj29 open reading frame. We searched for SNPs in Sj29 coding region by sequencing RT-PCR products after cloning into the pBlu $2 \mathrm{KSM}$ vector. The cDNA of 150 different individual worms were synthesized. The complete $576 \mathrm{bp} \mathrm{Sj} 29$ ORF was amplified by RT-PCR. The resulting RT-PCR products were sequenced directly or sequenced after being inserted into vectors. Sequence chromatogram traces of RT-PCR-amplified products were used to determine homozygote or heterozygote. For every homozygote, three positive bacteria clones were sequenced, while for every heterozygote, three or more clones were sequenced until both maternal and paternal alleles were sequenced. The raw sequence data were aligned using Mega 4 software ${ }^{15}$. DnaSP software ${ }^{16}$ was used to calculate $\pi$ : an estimate of the average number of nucleotide substitutions between any 2 sequences; S: the number of polymorphic sites in the sample; and A: the number of alleles. The Neighbor-joining tree of Sj29 alleles and Sm29 (GenBank Accession No.AF029222) based on $p$, the proportion of amino acid difference was generated by Mega 4 software. MacClade 4.08 was used for cladistic analysis (http://macclade.org).

Test of Neutrality. These rates of substitution within species were estimated using the method of Nei and Gojobori with the Jukes and Cantor correction ${ }^{17}$ as implemented in the MEGA 4 software. The rates of nonsynonymous and synonymous substitutions were compared with the Z-test of selection (MEGA 4).

Recombinant proteins preparation. Three majority alleles of $\mathrm{Sj} 29$ were selected for protein expression, $\mathrm{Sj} 29-2$, grouped in predominant population $\mathrm{A} ; \mathrm{Sj} 29-4$, grouped in predominant population $\mathrm{B}$; and $\mathrm{Sj} 29-20$, grouped in predominant population $\mathrm{C}$. The amino acid sequences of extracellular domain (Phe27-Lys170) were back-translated into DNA sequences using codon usage frequencies for the yeast Pichia pastoris. The recombiant proteins were expressed and purified as reported previously ${ }^{18}$.

Vaccination and infection. Mice ( $n=10$ per group) were injected subcutaneously with $20 \mu$ g of single recombinant protein (Sj29-2, Sj29-4 or Sj29-20) or antigen mixture $(6.7 \mu \mathrm{g}$ of each protein) in $30 \mu \mathrm{l}$ PBS formulated with $70 \mu \mathrm{l}$ ISA $70 \mathrm{M}$ (SEPPIC, France) three times at 3-week intervals. PBS group and $70 \mathrm{M}$ adjuvant group were set as control groups. On week 9 , mice were challenged with cercariae of Guichi field isolate or a labaratory line Sj6M8. Each mouse was infected with 40 cercariae. On week 15, adult worms were recovered by perfusion from the mesenteric vein and separated. The level of protection of vaccinated groups was calculated as a percentage based on the reduction in worm burdens or liver eggs per gram of liver, compared with those from the control group.

ELISA. Serum samples were collected three weeks after the third immunization. Enzyme-linked immunosorbent assay (ELISA) was performed to detect the antibody levels of IgG against Sj29-2, Sj29-4 or Sj29-20. ELISA was performed as described before $^{18}$. Cut-off values were determined from the mean plus three standard deviations from the pre-immunization sera.

Genotype of individual worm. We selected the trimorphic nucleotide site 162 to determine worm genotype, $\mathrm{C} 162 \mathrm{C}$ for genotype AA, G162G for genotype BB, A162A for genotype MM, C162G for genotype $\mathrm{AB}, \mathrm{C} 162 \mathrm{~A}$ for genotype $\mathrm{AM}$ and G162A for genotype BM (Supplementary Fig. 7). 
Statistic Analysis. Data of worm burdens and liver egg burdens were expressed as the mean \pm standard error. Multiple comparisons were analyzed by a LSD one-way ANOVA test, with a $p$-value $<0.05$ considered significant. The genotype frequency of survival worms in different groups was calculated, respectively. For statistic analysis, it should be better to use Kruskal Wallis test. However, since the number of survival worms from individual mice was very small and quite variable from 2 to 33 , we were not able to further categorize the survival worms with various genotypes and rank the data. Therefore, we pooled the survival worms of each group (10 mice) for Sj29 genotyping and used chi-square test or Fisher's exact test for analyzing the ratio of various genotypes. To increase the validity of our conclusion, we repeated the experiments including use of the antigen with different alleles. For Sj29-mix immunized group (Fig. 2D), considering that the mixture of the antigens induce immunity that killed both homozygotes and heterozygotes, which led to significant reduction of total worms and equal-ratio reduction of worms with various genotypes, we converted the data before the statistic analysis. Firstly we calculated the percentage of worms with various genotypes in PBS control group using the formula: e.g. $\mathrm{AB}$ genotype worms $\%=\mathrm{AB}$ genotype worms/total worms (i.e. $49 / 252=19.4 \%$ ). Then we used the percentage to calculate the number of the infected cercariae with various genotypes. Since we used 400 cercariae for infection of 10 mice each group ( 40 cercariae per mouse), the number of the infected $\mathrm{AB}$ genotype cercariae is $19.4 \% \times$ $400=78$. Thus, of 78 infected cercariae, the survival AB genotype worms were 49,41 , and 15 in the PBS, adjuvant and Sj29-mix groups, respectively. The ratios of survival worms in various groups were further compared by chi-square test.

Prediction Model. We established a predcition model to study the change of the frequencies of vaccine targeted alleles (clade A and B alleles) and untargeted alleles ("clade" $\mathrm{M}$ alleles) during period of 10 schistosome transmission seasons. We consider clades $\mathrm{A}, \mathrm{B}$ and $\mathrm{M}$, with frequencies $\mathrm{a}_{1}, \mathrm{~b}_{1}$ and $\mathrm{m}_{1}$, respectively. According to Hardy-Weinberg equilibrium, the frequencies of six different genotypes AA, BB, $M M, A B, A M$ and $B M$ are $a_{1}^{2}, b_{1}^{2}, m_{1}^{2}, 2 a_{1} b_{1}, 2 a_{1} m_{1}$ and $2 b_{1} m_{1}$. We consider the survival rates of vaccine targeted genotypes $\mathrm{AA}, \mathrm{BB}$ and $\mathrm{AB}$ as $\alpha, \beta$ and $\gamma$, respectively. In the survival population, the frequencies of clades $A, B$ and $M$ will be changed and can be calculated as the following formulas:

$\mathrm{a}_{\mathrm{i}}=\left(\mathrm{a}_{\mathrm{i}-1}^{2} \alpha+\mathrm{a}_{\mathrm{i}-1} \mathrm{~b}_{\mathrm{i}-1} \gamma+\mathrm{a}_{\mathrm{i}-1} \mathrm{~m}_{\mathrm{i}-1}\right) /\left(\mathrm{a}_{\mathrm{i}-1}^{2} \alpha+\mathrm{b}_{\mathrm{i}-1}^{2} \beta+\mathrm{m}_{\mathrm{i}-1}^{2}+2 \mathrm{a}_{\mathrm{i}-1} \mathrm{~b}_{\mathrm{i}-1} \gamma+2 \mathrm{a}_{\mathrm{i}-1} \mathrm{~m}_{\mathrm{i}-1}+2 \mathrm{~b}_{\mathrm{i}-1} \mathrm{~m}_{\mathrm{i}-1}\right)$ $\mathrm{b}_{\mathrm{i}}=\left(\mathrm{b}_{\mathrm{i}-1}^{2} \beta+\mathrm{a}_{\mathrm{i}-1} \mathrm{~b}_{\mathrm{i}-1} \gamma+\mathrm{b}_{\mathrm{i}-1} \mathrm{~m}_{\mathrm{i}-1}\right) /\left(\mathrm{a}_{\mathrm{i}-1}^{2} \alpha+\mathrm{b}_{\mathrm{i}-1}^{2} \beta+\mathrm{m}_{\mathrm{i}-1}^{2}+2 \mathrm{a}_{\mathrm{i}-1} \mathrm{~b}_{\mathrm{i}-1} \gamma+2 \mathrm{a}_{\mathrm{i}-1} \mathrm{~m}_{\mathrm{i}-1}+2 \mathrm{~b}_{\mathrm{i}-1} \mathrm{~m}_{\mathrm{i}-1}\right)$; $\mathrm{m}_{\mathrm{i}}=\left(\mathrm{m}_{\mathrm{i}-1}^{2}+\mathrm{a}_{\mathrm{i}-1} \mathrm{~m}_{\mathrm{i}-1}+\mathrm{b}_{\mathrm{i}-1} \mathrm{~m}_{\mathrm{i}-1}\right) /\left(\mathrm{a}_{\mathrm{i}-1}^{2} \alpha+\mathrm{b}_{\mathrm{i}-1}^{2} \beta+\mathrm{m}_{\mathrm{i}-1}^{2}+2 \mathrm{a}_{\mathrm{i}-1} \mathrm{~b}_{\mathrm{i}-1} \gamma+2 \mathrm{a}_{\mathrm{i}-1} \mathrm{~m}_{\mathrm{i}-1}+2 \mathrm{~b}_{\mathrm{i}-1} \mathrm{~m}_{\mathrm{i}-1}\right)$. In this study, $\mathrm{a}_{1}=0.55, \mathrm{~b}_{1}=0.35, \mathrm{~m}_{1}=0.13, \alpha=0.32, \beta=0.58, \gamma=0.37$, and thus we predicted the variation tendency of $\mathrm{a}_{\mathrm{i}}, \mathrm{b}_{\mathrm{i}}$ and $\mathrm{m}_{\mathrm{i}}$.

1. McManus, D. P. \& Loukas, A. Current status of vaccines for schistosomiasis. Clin Microbiol Rev 21, 225-242 (2008).

2. Loukas, A., Tran, M. \& Pearson, M. S. Schistosome membrane proteins as vaccines. Int J Parasitol 37, 257-263 (2007).

3. Bergquist, N. R., Leonardo, L. R. \& Mitchell, G. F. Vaccine-linked chemotherapy: can schistosomiasis control benefit from an integrated approach? Trends Parasitol 21, 112-117 (2005).

4. Tran, M. H. et al. Tetraspanins on the surface of Schistosoma mansoni are protective antigens against schistosomiasis. Nat Med 12, 835-840 (2006)

5. Cardoso, F. C. et al. Schistosoma mansoni Tegument Protein Sm29 Is Able to Induce a Th1-Type of Immune Response and Protection against Parasite Infection. Plos Neglect Trop Dis 2, e308 (2008).

6. Liu, F. et al. New perspectives on host-parasite interplay by comparative transcriptomic and proteomic analyses of Schistosoma japonicum. PLoS Pathog 2, e29 (2006).

7. Cai, P. et al. Molecular characterization of Schistosoma japonicum tegument protein tetraspanin-2: sequence variation and possible implications for immune evasion. Biochem Biophys Res Commun 372, 197-202 (2008).
8. Chen, H. et al. Immune protection of tegument protein $\mathrm{rSj} 29$ against Schistosoma japonicum in mice. Zhongguo Ji Sheng Chong Xue Yu Ji Sheng Chong Bing Za Zhi 27, 476-482 (2009).

9. Zhang, W. et al. Inconsistent protective efficacy and marked polymorphism limits the value of Schistosoma japonicum tetraspanin-2 as a vaccine target. PLoS Negl Trop Dis 5, e1166 (2011).

10. Takala, S. L. et al. Dynamics of polymorphism in a malaria vaccine antigen at a vaccine-testing site in Mali. Plos Med 4, 523-534 (2007).

11. Genton, B. et al. A recombinant blood-stage malaria vaccine reduces Plasmodium falciparum density and exerts selective pressure on parasite populations in a phase 1-2b trial in Papua New Guinea. J Infect Dis 185, 820-827 (2002).

12. Gaschen, B. et al. Diversity considerations in HIV-1 vaccine selection. Science 296, 2354-2360 (2002).

13. Escalante, A. A., Lal, A. A. \& Ayala, F. J. Genetic polymorphism and natural selection in the malaria parasite Plasmodium falciparum. Genetics 149, 189-202 (1998).

14. Polley, S. D. \& Conway, D. J. Strong diversifying selection on domains of the Plasmodium falciparum apical membrane antigen 1 gene. Genetics 158, 15051512 (2001).

15. Tamura, K., Dudley, J., Nei, M. \& Kumar, S. MEGA4: Molecular evolutionary genetics analysis (MEGA) software version 4.0. Mol Biol Evol 24, 1596-1599 (2007).

16. Rozas, J., Sanchez-DelBarrio, J. C., Messeguer, X. \& Rozas, R. DnaSP, DNA polymorphism analyses by the coalescent and other methods. Bioinformatics 19, 2496-2497 (2003).

17. Nei, M. \& Gojobori, T. Simple methods for estimating the numbers of synonymous and nonsynonymous nucleotide substitutions. Mol Biol Evol 3, 418426 (1986).

18. Xu, X. D. et al. A Schistosoma japonicum chimeric protein with a novel adjuvant induced a polarized Th1 immune response and protection against liver egg burdens. Bmc Infect Dis 9, e54 (2009).

\section{Acknowledgements}

We thank Drs Charles F. Delwiche at University of Maryland at College Park and Michael Dean at National Cancer Institute for their comments and discussion. This work is supported by grants from the National Basic Research Program (973 Program) in China (2007CB513100). Institutions 1 and 2 on the affiliation list contributed equally to this work.

\section{Author contributions}

X.X. and W.P. conceived and designed this study. X.X., J.S., J.Z., D.W. and X.Q. performed the experiments. X.X., J.Z. T.M. and W.P. participated in the statistical analysis. X.X., T.M. and W.P. drafted the manuscript. All authors contributed to the interpretation of the study.

\section{Additional information}

Supplementary information accompanies this paper at http://www.nature.com/ scientificreports

Competing financial interests: The authors declare no competing financial interests.

License: This work is licensed under a Creative Commons Attribution-NonCommercial-ShareAlike 3.0 Unported License. To view a copy of this license, visit http://creativecommons.org/licenses/by-nc-sa/3.0/

How to cite this article: Xu, X. et al. Having a pair: the key to immune evasion for the diploid pathogen Schistosoma japonicum. Sci. Rep. 2, 346; DOI:10.1038/srep00346 (2012). 